\title{
Study of Practical Effectiveness for Machine Translation Using Recursive Chain-link-type Learning
}

\author{
Hiroshi Echizen-ya \\ Dept. of Electronics and Information \\ Hokkai-Gakuen University \\ S 26-Jo, W 11-Chome, Chuo-ku \\ Sapporo, 064-0926 Japan \\ echi@eli.hokkai-s-u.ac.jp \\ Yoshio Momouchi \\ Dept. of Electronics and Information \\ Hokkai-Gakuen University \\ S 26-Jo, W 11-Chome, Chuo-ku \\ Sapporo, 064-0926 Japan \\ momouchi@eli.hokkai-s-u.ac.jp
}

\author{
Kenji Araki \\ Division of Electronics and Information \\ Hokkaido University \\ N 13-Jo, W 8-Chome, Kita-ku \\ Sapporo, 060-8628 Japan \\ araki@media.eng.hokudai.ac.jp \\ Koji Tochinai \\ Division of Business Administration \\ Hokkai-Gakuen University \\ 4-Chome, Asahi-machi, Toyohira-ku \\ Sapporo, 060-8790 Japan \\ tochinai@econ.hokkai-s-u.ac.jp
}

\begin{abstract}
A number of machine translation systems based on the learning algorithms are presented. These methods acquire translation rules from pairs of similar sentences in a bilingual text corpora. This means that it is difficult for the systems to acquire the translation rules from sparse data. As a result, these methods require large amounts of training data in order to acquire high-quality translation rules. To overcome this problem, we propose a method of machine translation using a Recursive Chain-linktype Learning. In our new method, the system can acquire many new high-quality translation rules from sparse translation examples based on already acquired translation rules. Therefore, acquisition of new translation rules results in the generation of more new translation rules. Such a process of acquisition of translation rules is like a linked chain. From the results of evaluation experiments, we confirmed the effectiveness of Recursive Chain-link-type Learning.
\end{abstract}

\section{Introduction}

Rule-Based Machine Translation(MT)(Hutchins and Somers, 1992) requires large-scale knowledge to analyze both source language(SL) sentences and target language(TL) sentences. Moreover, it is difficult for a developer to completely describe large-scale knowledge that can analyze various linguistic phenomena. Therefore, Rule-Based MT is time-consuming and expensive. Statistical MT and Example-Based
MT have been proposed to overcome the difficulties of Rule-Based MT. These approaches correspond to Corpus-Based approach. CorpusBased approach uses translation examples that keep including linguistic knowledge. This means that the system can improve the quality of its translation only by adding new translation examples. However, in Statistical MT(Brown et al., 1990), large amounts of translation examples are required in order to obtain high-quality translation. Moreover, ExampleBased MT(Sato and Nagao, 1990; Watanabe and Takeda, 1998; Brown, 2001; Carl, 2001) which relies on various knowledge resources results in the same difficulties as Rule-Based MT. Therefore, Example-Based MT, which automatically acquires the translation rules from only bilingual text corpora, is very effective. However, existing Example-Based MT systems using the learning algorithms require large amounts of translation pairs to acquire high-quality translation rules.

In Example-Based MT based on analogical reasoning(Malavazos, 2000; Guvenir, 1998), the different parts are replaced by variables to generalize translation examples as shown in (1) of Figure 1. However, the number of different parts of the two SL sentences must be same as the number of different parts of the two TL sentences. This means that the condition of acquisition of translation rules is very strict because this method allows only $n: n$ mappings in the number of the different parts between the 
SL sentences and the TL sentences. As a result, many translation rules cannot be acquired. (McTait, 2001) generalizes both the different parts and the common parts as shown in Figure 1(2). This means that (McTait, 2001) allows $m: n$ mappings in the number of the different parts, or the number of the common parts. However, it is difficult to acquire the translation rules that correspond to the lexicon level. On the other hand, we have proposed a method of Machine Translation using Inductive Learning with Genetic Algorithms(GA-ILMT)(Echizenya et al., 1996). This method automatically generates the similar translation examples from only given translation examples by applying genetic algorithms(Goldberg, 1989) as shown in (3a) of Figure 1. Moreover, the system performs Inductive Learning. By using Inductive Learning, the abstract translation rules are acquired by performing phased extraction of different parts as shown in Figure 1(3b) and (3c). In all methods shown in Figure 1, the condition of acquisition of translation rules is that two similar translation examples must exist. As a result, the systems require large amounts of translation examples.

We propose a method of MT using Recursive Chain-link-type Learning as a method to overcome the above problem. In our method, the system acquires new translation rules from sparse data using other already acquired translation rules. For example, first, translation rule $\mathrm{B}$ is acquired by using translation rule $\mathrm{A}$ when the translation rule A exists in the dictionary. Moreover, translation rule $\mathrm{C}$ is acquired by using the translation rule B. Such a process of acquisition of translation rules is like a chain where each ring is linked. Therefore, we call this mechanism Recursive Chain-link-type Learning(RCL). This method can effectively acquire many translation rules from sparse data without depending on the different parts of similar translation pairs. In this paper, we describe the effectiveness of RCL through evaluation experiments.

\section{Basic Idea}

RCL is a method with an ability that automatically acquires translation knowledge in a computer without any analytical knowledge, such as GA-ILMT. This is the ability to extract corre-
(1) Generalization of Different Parts (Güvenir and Cicekli, 1998)

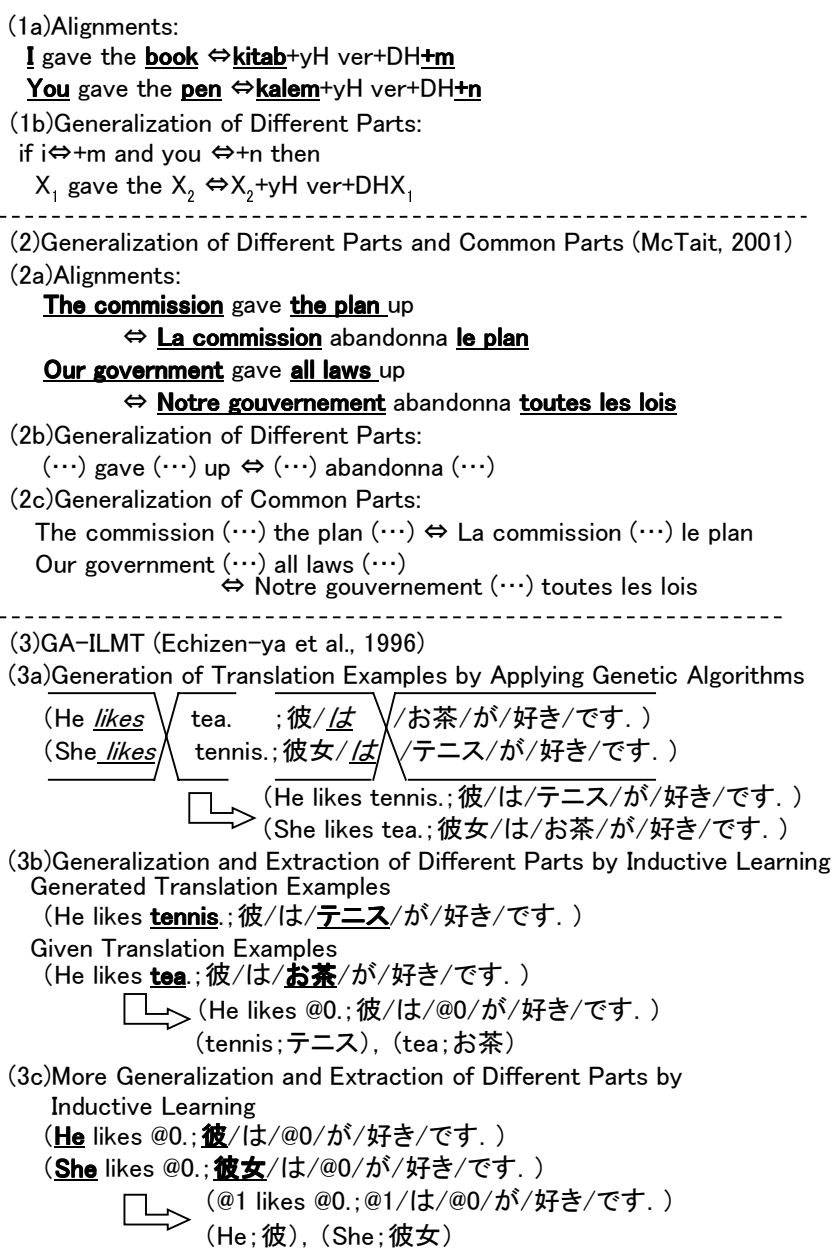

Figure 1: Previous works.

sponding parts from pairs of objects with which it corresponds. In this paper, we apply this ability to a translation example that consists of SL and TL sentences. A system with RCL can acquire translation rules from sparse translation examples. Figure 2 shows how translation rules are acquired using this method ${ }^{1}$.

Figure 2 shows the process where translation rules $\mathrm{B}, \mathrm{C}$ and $\mathrm{D}$ are acquired one after another using RCL. In this paper, source parts are those parts that are extracted from the SL sentences of translation examples, and target parts are those parts that are extracted from the TL sentences of translation examples. Moreover, part translation rules are pairs of source parts and

\footnotetext{
${ }^{1}$ In Figure 2, the use of a Greek character means that all language characters correspond to unknown character strings for a computer.
} 


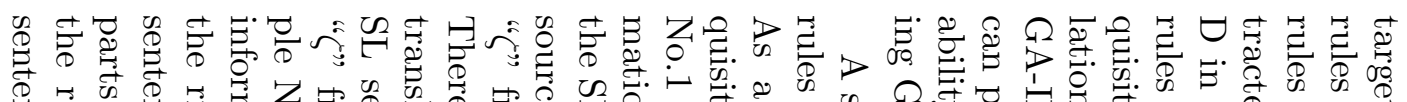

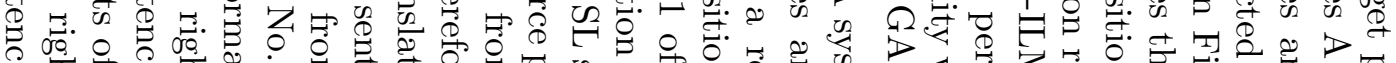

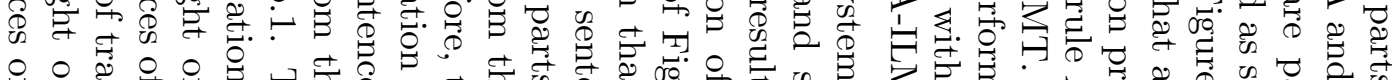

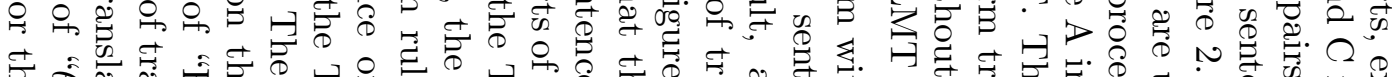
。

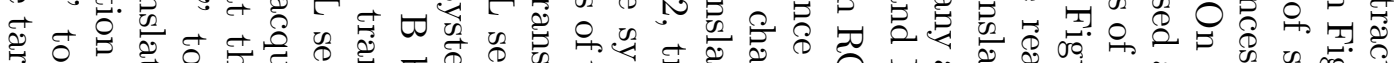

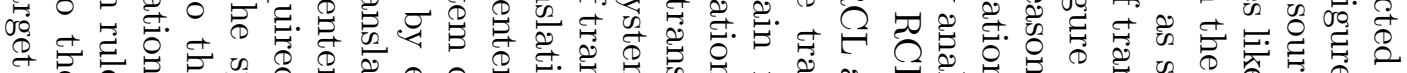
to 00

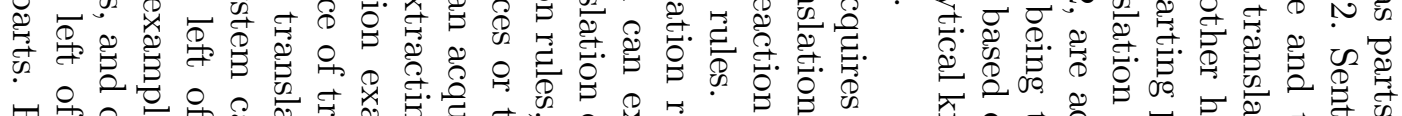

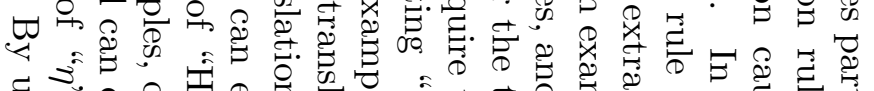
है.

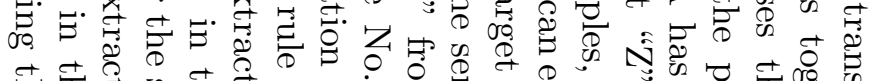

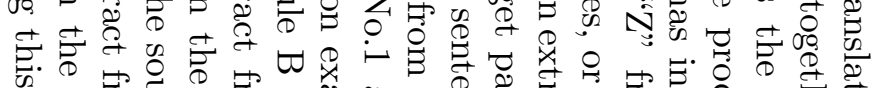

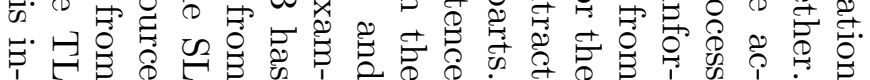

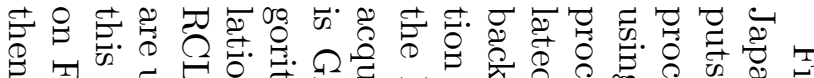

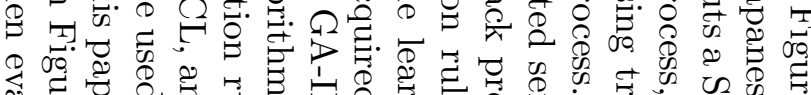

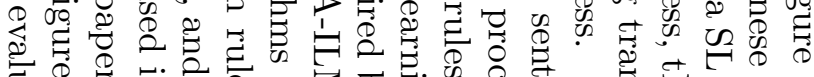

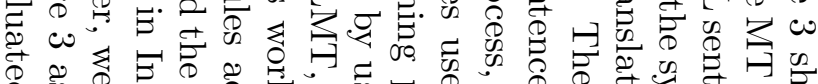
$\Sigma$ \&

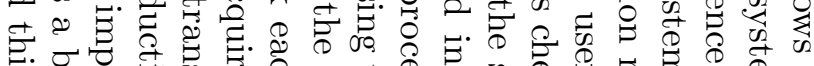
ज. की

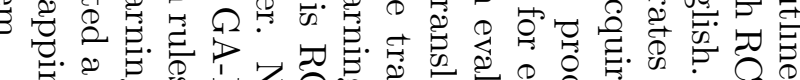

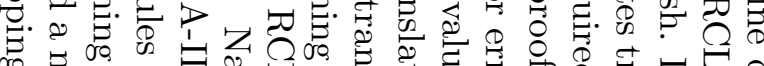

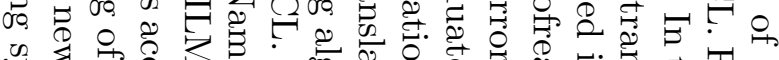

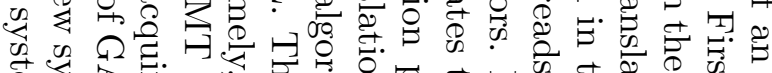

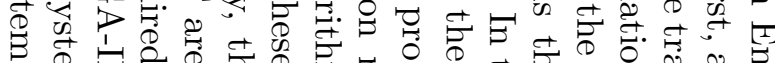

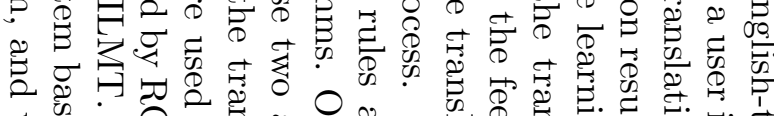

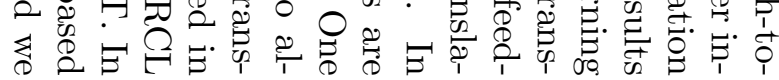
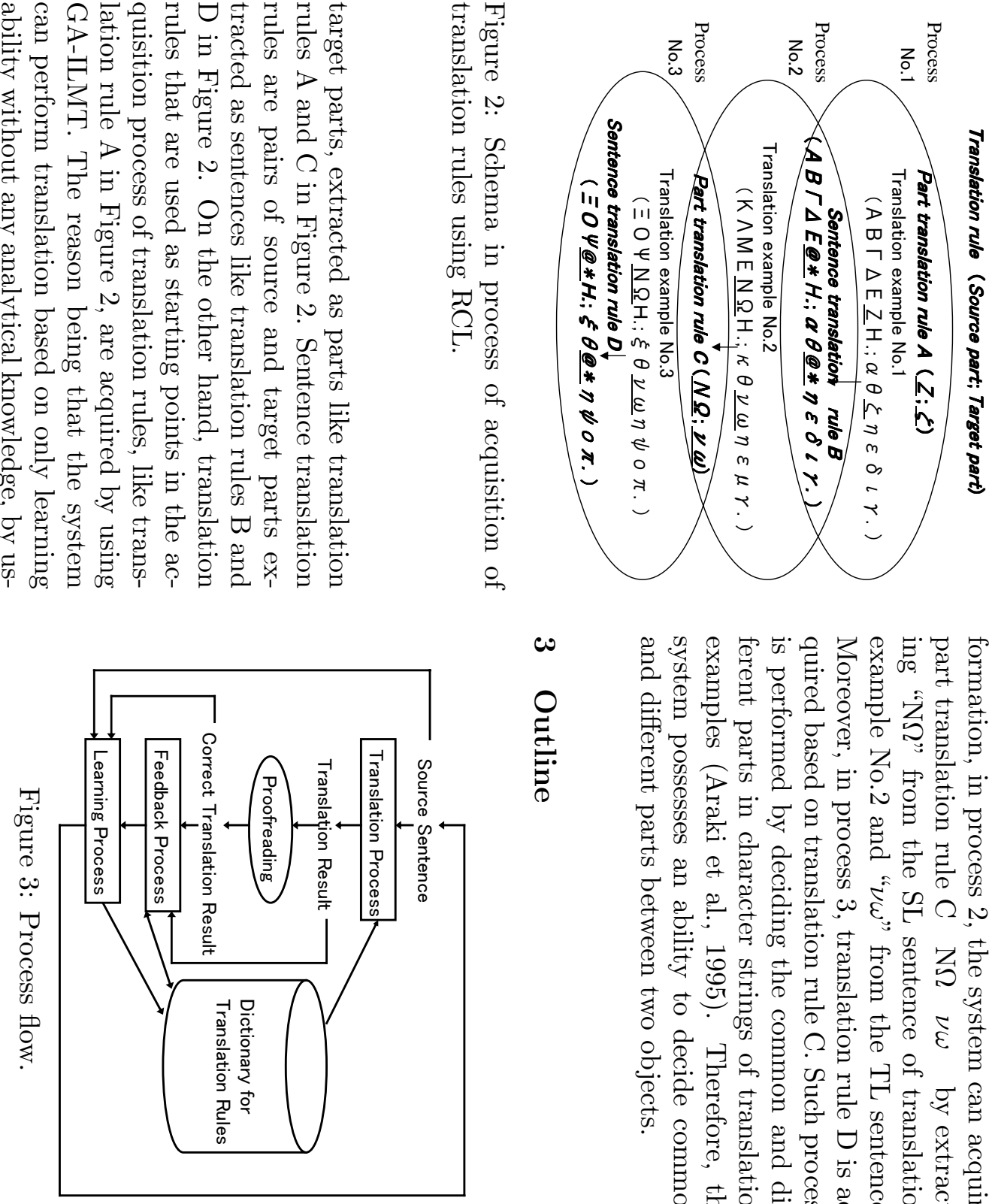

$\omega$

:

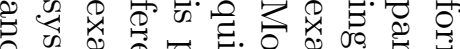

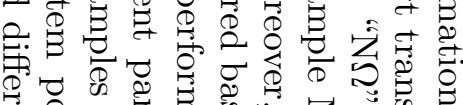

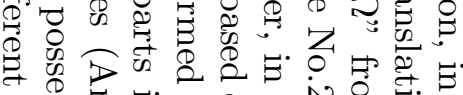

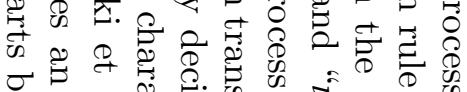
ब

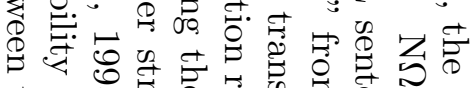

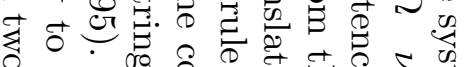

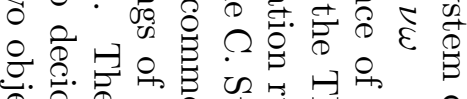

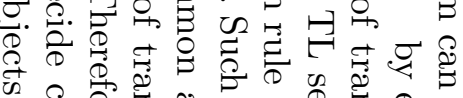
क 800

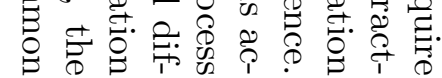

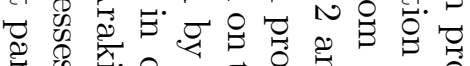




\section{Process}

\subsection{Translation process}

In the translation process, the system generates translation results using acquired translation rules. First, the system selects the sentence translation rules that can be applied to the SL sentence. Second, the system generates the translation results by replacing the variables in the sentence translation rules with the part translation rules.

\subsection{Feedback process}

In the feedback process, the system evaluates the translation rules used. First, the system evaluates the translation rules without variables by using the results of combinations between the translation rules with variables and the translation rules without variables(Echizen-ya et al., 1996). Next, the system evaluates translation rules with variables by using the processes of combinations between the translations rules with variables and the translation rules without variables(Echizen-ya et al., 2000). As a result, the system increases the correct translation frequencies, or the erroneous translation frequencies, of the translation rules by using these evaluation methods for the translation rules.

\subsection{Learning process}

\subsubsection{GA-ILMT}

In this paper, by using the process of acquisition of translation rules in GA-ILMT, the system acquires both sentence and part translation rules. These rules are then used as starting points when the system performs RCL.

\subsubsection{Recursive Chain-link-type Learning(RCL)}

In this section, we describe the process of acquisition of translation rules using RCL. The details of the process of acquisition of part translation rules are as follows.

(1)The system selects translation examples that have common parts with the sentence translation rules.

(2)The system extracts the parts that correspond to the variables in the source parts and in the target parts of the sentence translation rules from the SL sentences, and the TL sentences of the translation examples.
(3) The system registers pairs, of the parts extracted from the SL sentences and the parts extracted from the TL sentences, as the part translation rules.

(4)The system gives the correct and erroneous frequencies of sentence translation rules to the acquired part translation rules.

Figure $4^{2}$ shows an example of the acquisition of a part translation rule using the sentence translation rule. In Figure 4, (thirty;30[sanju]) as the part translation rule is acquired because "thirty" corresponds to the variable in the source part of sentence translation rule and "30[sanju]" corresponds to the variable in the target part of sentence translation rule.

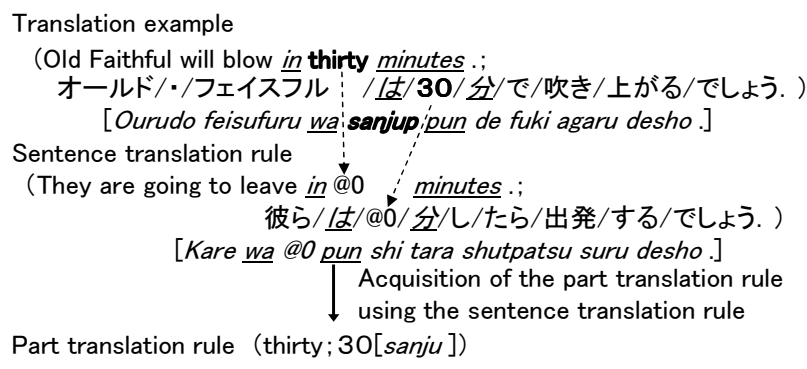

Figure 4: Example of the acquisition of a part translation rule using the sentence translation rule.

The details of the process of acquisition of sentence translation rules are as follows:

(1)The system selects the part translation rules in which the source parts are included in the SL sentences of the translation example or in the source parts of sentence translation rules, and in which the target parts are included in the TL sentences of the translation examples or in the target parts of sentence translation rules.

(2)The system acquires new sentence translation rules by replacing the parts which are same as the part translation rules with the variables to the translation examples or the sentence translation rules.

(3) The system gives the correct and erroneous frequencies of the part translation rules to the acquired sentence translation rules.

\footnotetext{
${ }^{2}$ Italics are the pronunciation in Japanese.
} 
Figure 5 shows examples of the acquisition of the sentence translation rules using the part translation rules. In Figure 5, the system acquires (It starts in @0 minutes. ; 乥れ/は /@0/分/たて/ば/始まり/ます.[Sorewa@0 pun tate ba hajimari masu.]) as a sentence translation rule by using the part translation rule (thirty;30[sanju]) acquired in Figure 4, and ( @1 starts in@0 minutes. ; @1/は/@0/分/たて/ば/ 始まり/ます.[@1wa@0 pun tateba hajimari masu.]) as the sentence translation rule, that is more abstracted, is acquired by using the part translation rule (it; 乥れ [sore]).

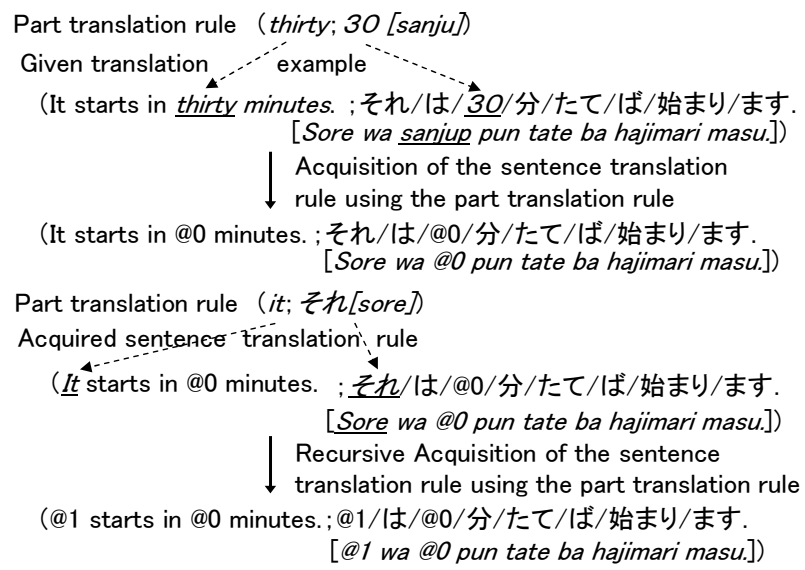

Figure 5: Examples of the acquisition of a sentence translation rule using the part translation rule.

\section{$5 \quad$ Experiments for performance evaluation}

\subsection{Experimental procedure}

There are two kinds of data as experimental data. One is learning data and the other is evaluation data. In these experiments, 1,759 translation examples were used as learning data. These translation examples were taken from textbooks(Nihon Kyozai(1), 2001; Nihon Kyozai(2), 2001; Hoyu Shuppan, 2001) for secondgrade junior high school students. As well, 1,097 translation examples were used as evaluation data. These translation examples were taken from textbooks(Bunri, 2001; Sinko Shuppan, 2001) for second-grade junior high school students. All of these translation examples were processed by the method outlined in Figure 3. The initial condition of the dictionary is empty. Moreover, we used three other commercial RuleBased MT systems, comparing our system with those systems. We call these three MT systems $\mathrm{A}, \mathrm{B}$ and $\mathrm{C}$ respectively.

\subsection{Evaluation standards}

The correct translation results are grouped into two categories:

(1) The correct translation

This means that the translation results correspond to the correct translation results taken from textbooks respectively(Bunri, 2001; Sinko Shuppan, 2001).

(2) A correct translation which includes unknown words

This means that the translation results with substituted nouns or adjectives as variables correspond to the correct translation results taken from textbooks respectively(Bunri, 2001; Sinko Shuppan, 2001).

In this paper, the effective translation results are the translation results that correspond to (1) and (2), and the ineffective translation results are the translation results that do not correspond to (1) and (2). Moreover, the effective translation rate is the rate of the effective translation results in all the evaluation data. The translation results are ranked when several translation results are generated. The translation results using the translation rules whose rate of correct translation frequency is high, are ranked at the top. We evaluated the translation results that are ranked from No.1 to No.3.

\subsection{Experimental results and discussion}

Table 1 shows examples of effective translation results in our system with RCL. Table 2 shows the results of comparative experiments of our system and the three Rule-Based MT systems. We excluded 309 SL sentences from 1,097 SL sentences used as evaluation data in Table 2 . In our system, the 309 SL sentences became the ineffective translation results because of a lack of learning data. Therefore, the 309 SL sentences are not inadequate as evaluation data. Table 2 shows the effective translation rates in 788 SL sentences, which were left after excluding 309 SL sentences from the 1,097 SL sentences used as evaluation data. In the other three RuleBased MT systems, the same 788 SL sentences were used as evaluation data and the translation results which correspond to (1) and (2) 
Table 1: Examples of effective translation results.

\begin{tabular}{|c|c|}
\hline \multicolumn{2}{|r|}{ Examples of the correct translation results } \\
\hline SL sentences & TL sentences \\
\hline This bag was made in France. & このバッグはフランス製です. Kono baggu wa furansu sei desu. \\
\hline $\begin{array}{l}\text { We went there to play } \\
\text { baseball. }\end{array}$ & $\begin{array}{l}\text { わたしたちは野球をするため宁こへ行きました . } \\
\text { [Watashi tachi wa yakyu wo suru tame soko e iki mashi ta.] }\end{array}$ \\
\hline \multicolumn{2}{|c|}{ Examples of the correct translation results which includes the unknown words } \\
\hline SL sentences & TL sentences \\
\hline $\begin{array}{l}\text { Shall I take you to the } \\
\text { amusement park? }\end{array}$ & $\begin{array}{l}\text { @0へ連れていってあげましようか? } \\
\text { [@0 e tsure te itte age masho ka?] } \\
\text { @0 requires "遊園地 [yuen chi]" which is equivalent for } \\
\text { the noun "the amusement park". }\end{array}$ \\
\hline $\begin{array}{l}\text { How far is it from Kyoto to } \\
\text { Hiroshima? }\end{array}$ & $\begin{array}{l}\text { @0 から広島までどのくらいの距離がありますか？[@0 kara } \\
\text { hiroshima made dono kurai no kyori ga ari masu ka?] } \\
\text { @0 requires “京都 [kyoto]" which is equivalent for } \\
\text { the noun "Kyoto". }\end{array}$ \\
\hline
\end{tabular}

described in section 5.2 were evaluated as the correct translation results. The effective translation rate in the system with only GA-ILMT was $45.1 \%$. In Table 2 , the effective translation rate of system with RCL is almost the same as the effective translation rates of system $\mathrm{A}$, but is higher than systems B and C.

Table 2: Results of comparative experiments.

\begin{tabular}{c|c|c|c}
\hline \multirow{2}{*}{ System } & Effective trans- & \multicolumn{2}{|c}{ Details } \\
\cline { 3 - 4 } & lation rates & $(1)$ & $(2)$ \\
\hline Our system & $85.0 \%$ & $41.6 \%$ & $58.4 \%$ \\
\hline system A & $85.8 \%$ & $84.0 \%$ & $16.0 \%$ \\
\hline system B & $81.7 \%$ & $83.7 \%$ & $16.3 \%$ \\
\hline system C & $76.9 \%$ & $82.7 \%$ & $17.3 \%$ \\
\hline
\end{tabular}

Table 3: Comparison of effective translation rates based on quality.

\begin{tabular}{c|c|c|c}
\hline \multirow{2}{*}{ System } & Effective trans- & \multicolumn{2}{|c}{ Details } \\
\cline { 3 - 4 } & lation rates & $(1)$ & $(2)$ \\
\hline Our system & $73.7 \%$ & $7.5 \%$ & $52.5 \%$ \\
\hline system A & $70.3 \%$ & $84.2 \%$ & $15.8 \%$ \\
\hline system B & $63.8 \%$ & $85.0 \%$ & $15.0 \%$ \\
\hline system C & $58.7 \%$ & $82.8 \%$ & $17.2 \%$ \\
\hline
\end{tabular}

Moreover, we evaluated translation results more strictly in terms of the quality of translation. Meaning that only translation results that had almost the same character strings as the correct translation results taken from the textbooks(Bunri, 2001; Sinko Shuppan, 2001) were effective translation results. For exam- ple，“乥れは約 10 分かかります [Sore wa yaku juppun kakari masu.]" is an ineffective translation result because of the correct translation results for "It takes about ten minutes." is “約 10 分かかります $[Y a k u$ juppun kakari masu.]" in textbook(Bunri, 2001; Sinko Shuppan, 2001). In this Japanese sentence, phrase “关れは [sore wa]" results in needlessly long. Therefore, we evaluate the translation results that have different phrases to the correct translation results as the ineffective translation results in terms of the quality of translation. Table 3 shows a comparison of effective translation rates based on quality. In Table 3 , we confirmed that the system with RCL can generate more high-quality translation results than the three other Rule-Based MT systems.

In the system with RCL, the erroneous translation rules are also acquired like a linked chain. For example, in Figure 2, the translation rules $\mathrm{B}, \mathrm{C}$ and $\mathrm{D}$ are acquired as the erroneous translation rules when the translation rule $\mathrm{A}$ is the erroneous translation rule. Namely, a chain reaction causes the acquisition of erroneous translation rules. In learning data, the rate of erroneous part translation rules to the acquired part translation rules was $47.9 \%$, and the rate of erroneous sentence translation rules to the acquired sentence translation rules was $38.2 \%$. However, such erroneous translation rules are automatically decided as being erroneous translation rules in the feedback process resulting from the ineffective translation results. 


\section{Conclusion}

In existing Example-Based MT systems based on learning algorithms, similar translation pairs must exist to acquire high-quality translation rules. This means that the systems require large amounts of translation examples to acquire high-quality translation rules. On the other hand, a system with RCL can acquire many new translation rules from sparse translation examples because it uses other already acquired translation rules based on the learning algorithms described in section 2 . As a result, the quality of the translation and the effective translation rate of our system is higher than other Rule-Based MT systems. However, our system still does not reach the level of a practical MT system and requires more translation rules to realize the goal of a practical MT system. Although our system is not a practical enough MT system, the system can effectively acquire the translation rules from sparse data by using RCL. Therefore, we consider that the quality of translation improves only by adding new translation examples without the difficulty of Rule-Based MT systems in which a developer must completely describe large-scale knowledge.

In the future, we plan to add a mechanism that effectively combines the acquired translation rules so that the system realizes the translation of practical SL sentences.

\section{Acknowledgements}

This work was partially supported by the Grants from the High-Tech Research Center of Hokkai-Gakuen University and a Government subsidy for aiding scientific research (No.14658097) of the Ministry of Education, Culture, Sports, Science and Technology of Japan.

\section{References}

Hutchins, W. J and H. L. Somers. 1992. An Introduction to Machine Translation. $A C A$ DEMIC PRESS.

Sato, S and M. Nagao. 1990. Toward Memorybased Translation. In proceedings of the Coling'90.

Brown, P., J. Cocke, S. Della Pietra, V. J. Della Pietra, F. Jelinek, J. D. Lafferty, R. L. Mercer and P. S. Roossin. 1990. A Statistical Approach to Machine Translation. Computational Linguistics Vol.16, No.2.
Watanabe, H and K. Takeda. 1998. A Patternbased Machine Translation System Extended by Example-based Processing. In proceedings of the Coling-ACL'98.

Brown, R.D. 2001. Transfer-Rule Induction for Example-Based Translation. In proceedings of the Workshop on EBMT, MT Summit VIII.

Carl, M. 2001. Inducing Translation Grammars from Bracketed Alignments. In proceedings of the Workshop on EBMT, MT Summit VIII.

Malavazos, C and S. Piperidis. 2000. Application of Analogical Modelling to Example Based Machine Translation. In proceedings of the Coling2000.

Güvenir, H.A and I. Cicekli. 1998. Learning Translation Templates from Examples. Information Systems Vol.23, No.6.

McTait, K. 2001. Linguistic Knowledge and Complexity in an EBMT System Based on Translation Patterns. In proceedings of the Workshop on EBMT, MT Summit VIII.

Echizen-ya, H., K. Araki, Y. Momouchi and K. Tochinai. 1996. Machine Translation Method using Inductive Learning with Genetic Algorithms. In proceedings of the Coling'96.

Goldberg, D. E. 1989. Genetic Algorithms in Search, Optimization, and Machine Learning. Addison-Wesley.

Araki, K., Y. Momouchi and K. Tochinai. 1995. Evaluation for Adaptability of Kana-kanji Translation of Non-segmented Japanese Kana Sentences using Inductive Learning. In proceedings of the PACLING'95.

Echizen-ya, H., K. Araki, Y. Momouchi and K. Tochinai. 2000 Effectiveness of Layering Translation Rules based on Transition Networks in Machine Translation using Inductive Learning with Genetic Algorithms. In proceedings of the MT and Multilingual Applications in the New Millennium.

Nihon-Kyozai(1). 2001. One World English Course 1 new edition. Tokyo.

Nihon-Kyozai(2). 2001. One World English Course 2 new edition. Tokyo.

Hoyu Shuppan. 2001. System English Course 2 new edition. Tokyo.

Bunri. 2001. Work English Course 2 new edition. Tokyo.

Sinko Shuppan 2001. Training English Course 2 new edition. Osaka. 